\title{
Lymph Node Ratio for Nodal Staging in Colorectal Cancer - a Promising, but Premature Tool
}

\author{
Kang Young Lee \\ Department of Surgery, Yonsei Universtiy College of Medicine, Seoul, Korea
}

\section{See Article on Page 252-265}

Accurate tumor staging is an essential step for the management of colorectal cancer patients. Precise tumor staging has a pivotal role not only for the prediction of the patient's prognosis, but also for the selection of patients for adjuvant therapy. The Tumor-node-metastasis (TNM) staging system based on pathologic findings is the most popular staging system in clinical practice.

Among several prognosticators in TNM staging, the presence of regional lymph-node metastasis is the most potent indicator of the patient's prognosis in cases of colorectal cancer. In the current staging system, nodal stage is classified by the number of metastatic lymph nodes. In addition, several studies have reported that the number of retrieved lymph nodes also has prognostic power because a sufficient number of lymph nodes should be examined for accurate staging. For that reason, in the American Joint Committee on Cancer (AJCC) 7th edition recommends that at least 10-14 lymph nodes in radical colon and rectum resections should be examined for reliable nodal staging [1].

The number of retrieved lymph nodes could be affected by the extent of surgery and the pertinacity of the pathologist, but the location of tumor, the application of preoperative radiotherapy, and patient factors, including body mass index, could also be contributing factors. Moreover, in cases of preoperative chemoradiation therapy, the proportion of those with fewer than 12 examined lymph nodes may be increased. Since a prerequisite for the current nodal staging is the examination of over

Correspondence to: Kang Young Lee, M.D.

Department of Surgery, Gangnam Severance Hospital Yonsei University College of Medicine, 146-92 Doguk-dong, Gangnam-gu, Seoul 135-720, Korea

Tel: +82-2-2019-3370, Fax: +82-2-3462-5994

E-mail: kylee117@yuhs.ac

(c) 2011 The Korean Society of Coloproctology

This is an open-access article distributed under the terms of the Creative Commons Attribution NonCommercial License (http://creativecommons.org/licenses/by-nc/3.0) which permits unrestricted noncommercial use, distribution, and reproduction in any medium, provided the original work is properly cited.
10-14 regional lymph nodes, nodal staging in stage III colorectal cancer, which is solely based on the number of metastatic lymph nodes, would be inaccurate in certain circumstances.

Recently, the metastatic lymph-node ratio has been suggested as an alternative tool for nodal staging in stage III colorectal cancer [2-4]. Also, after preoperative chemoradiation therapy in rectal cancer patients, the lymph-node ratio has reportedly been able to provide better stratification of patients' prognoses than ypN staging [5]. A recent series of publications support the lymph-node ratio's being a successful substitute for the current nodal staging in overcoming the limitation of the numeric counting system in cases of colorectal cancer. However, there are still several problems in the studies regarding the lymphnode ratio.

The biggest hurdle in nodal staging with the lymph-node ratio may be that there is no consensus on a point of reference for stratification. Even though the lymph-node ratio has shown very nice stratification of patients' prognoses in cases of stage III colorectal cancer, not all studies adapted the same point of reference. Standardization of the point of reference would be an essential step toward use of the lymph-node ratio in staging. There is no doubt that precise lymph-node staging would be an essential step toward providing accurate prognoses for colorectal cancer patients and selecting candidates for adjuvant therapy. Several studies have demonstrated the possibility of using the lymph-node ratio as a tool for more accurate nodal staging in colorectal cancer. However, many obstacles remain to be overcome before the acceptance of the lymph-node ratio in our daily practice.

\section{REFERENCES}

1. Sobin LH, Gospodrowicz MK, Wittekind C. TNM classification of malignant tumors (UICC International Union Against Cancer). 7th ed. New York: Wiley-Blacvkwell; 2009.

2. Peschaud F, Benoist S, Julie C, Beauchet A, Penna C, Rougier P, et al. The ratio of metastatic to examined lymph nodes is a powerful independent prognostic factor in rectal cancer. Ann Surg 2008; 248:1067-73. 
3. Rosenberg R, Friederichs J, Schuster T, Gertler R, Maak M, Becker $\mathrm{K}$, et al. Prognosis of patients with colorectal cancer is associated with lymph node ratio: a single-center analysis of 3026 patients over a 25-year time period. Ann Surg 2008;248:968-78.

4. Moug SJ, Saldanha JD, McGregor JR, Balsitis M, Diament RH.
Positive lymph node retrieval ratio optimizes patient staging in colorectal cancer. Br J Surg 2009;100:1530-3.

5. Kang J, Hur H, Min BS, Lee KY, Kim NK. Prognostic impact of the lymph node ratio in rectal cancer patients who underwent preoperative chemoradiation. J Surg Oncol 2011;104:53-8. 